\title{
SOLID DISPERSION: A FRUITFUL APPROACH FOR IMPROVING THE SOLUBILITY AND DISSOLUTION RATE OF POORLY SOLUBLE DRUGS
}

\author{
*Tyagi Reena, Dhillon Vandana \\ Department of Pharmacy, Lloyd Institute of Management \& Technology, Plot - 11, Greater Noida, U. P., INDIA \\ *Corresponding Author's E-mail ID-vandanadhillon@gmail.com, Mobile No. +919999708884
}

Received 12 June 2012; Review Completed 13 July 2012; Accepted 13 July 2012, Available online 15 July 2012

\begin{abstract}
ABS TRACT
In recent years, the formulation of poorly soluble compounds presented interesting challenges for formulation scientists in the pharmaceutical industry. Up to $40 \%$ of new chemical entities discovered by the pharmaceutical industry are poorly soluble or lipophilic compounds, which lead to poor oral bioavailability, high intra and inter subject variability and lack of dose proportionality. This frequently results in potentially important products not reaching the market or not achieving their full potential. Poorly water-soluble drugs often require high doses in order to reach therapeutic plasma concentrations after oral administration. Improvement in the extent and rate of dissolution is highly desirable for such compounds, as this can lead to an increased and more reproducible oral bioavailability and subsequently to clinically relevant dose reduction and more reliable therapy. The enhancement of dissolution rate and oral bioavailability is one of the greatest challenges in the development of poorly water soluble drugs. Experience with solid dispersions over the last 20-30 years indicates that this is a very fruitful approach to improving the solubility, dissolution rates and consequently the bioavailability of poorly soluble drugs. Solid dispersion is used to produce a homogeneous distribution of a small amount of drug in solid state. This article summarize some of the practical aspects for the preparation of solid dispersion like selection of carrier ,method of physicochemical characterization, their advantages, limitations and applications along with an insight into the molecular arrangement of drug in solid dispersion. Solid dispersion technique is a very useful method for pharmaceutical point of view because of its capability to solve the solubility problems by using solid dispersion method.
\end{abstract}

Key words: Solid dispersion, solubility, dissolution, selection of carriers

\section{INTRODUCTION ${ }^{1}$}

More than $90 \%$ of drugs are approved since 1995 have poor solubility. It is estimated that $40 \%$ of active new chemical entities (NCEs) identified in combinatorial screening programs employed by many pharmaceutical companies are poorly water soluble ${ }^{1}$. Drug absorption, sufficient and reproducible bioavailability and/or pharmacokinetic profile in humans are recognized today as one of the major challenges in oral delivery of new drug substances. Orally administered drugs on the Model list of Essential Medicines of the World Health Organization (WHO) are assigned BCS classifications on the basis of data available in the public domain. Of the 130 orally administered drugs on the WHO list, 61 could be classified with certainty. $84 \%$ of these belong to class I (highly soluble, highly permeable), $17 \%$ to clas s II (poorly soluble, highly permeable), 24 (39\%) to clas S III (highly soluble, poorly permeable) and $6(10 \%)$ to class IV (poorly soluble, poorly permeable). The rate and extent of absorption of class II \& class IV compounds is highly dependent on the bioavailability which ultimately depends on solubility ${ }^{2}$. Due to this major reason, solubility and dissolution rate enhancement is one of the important parameters which should be considered in formulation development of orally administered drug with poor aqueous solubility ${ }^{3}$.

A poorly water soluble drug, more recently, has been defined in general terms as a drug which requires more time to dissolve in the gastrointestinal fluid than it may take to get absorbed in the gastrointestinal tract and thus the ability to deliver poorly soluble drugs will grow in significance in the coming years as NCEs are relied upon for a larger share of the revenue within the pharmaceutical market by innovator companies. Similarly, generic drug manufacturers will need to employ economically efficient methods of delivery as more low solubility drugs go off patent, in order to maintain a competitive edge and sufficiently compete as profit margins shrink in this pricesensitive industry ${ }^{4}$.

Orally administered drugs should undergo dissolution in the gastro-intestinal fluids before absorption can commence. Insoluble or poorly soluble drugs are generally poorly absorbed from the gastro-intestinal tract after oral adminis tration. The absorption behavior of such drugs can be best studied by in-vitro dis solution characteristics ${ }^{6}$.

Dissolution rate of drug plays a vital role in absorption of the drug from the dosage form and hence its bioavailability. When a drug is admin istered orally in solid dosage form (such as tablets or capsules), it is designed to undergo series of predetermined stages. The first step towards the absorption process is the disintegration of the dosage form. The second and in fact the slowest or ratelimiting step is found to be dissolution of drug in the fluid at the absorption site (Figure - 2). When dissolution is the controlling step in the overall process, absorption is said to be dissolution rate limited. Since the rate-limiting step in the absorption process is dissolution of drug, therefore, any factor influencing the rate of dissolution must also influence the rate of absorption ${ }^{7}$. 


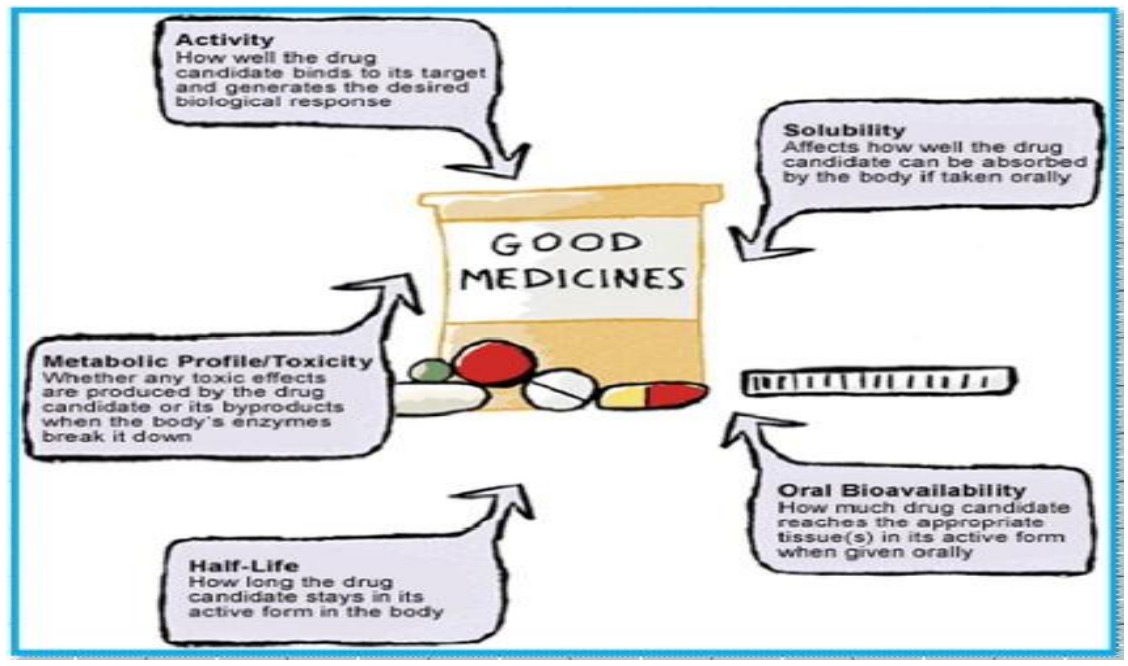

Figure 1: Good Medicines ${ }^{5}$

According to dissolution theory, two important parameters determining the dissolution rate of a solid in a given solvent are:
1) The solubility of the drug in the dissolution mediu mand

2) The surface area of the drug exposed to the dissolution medium

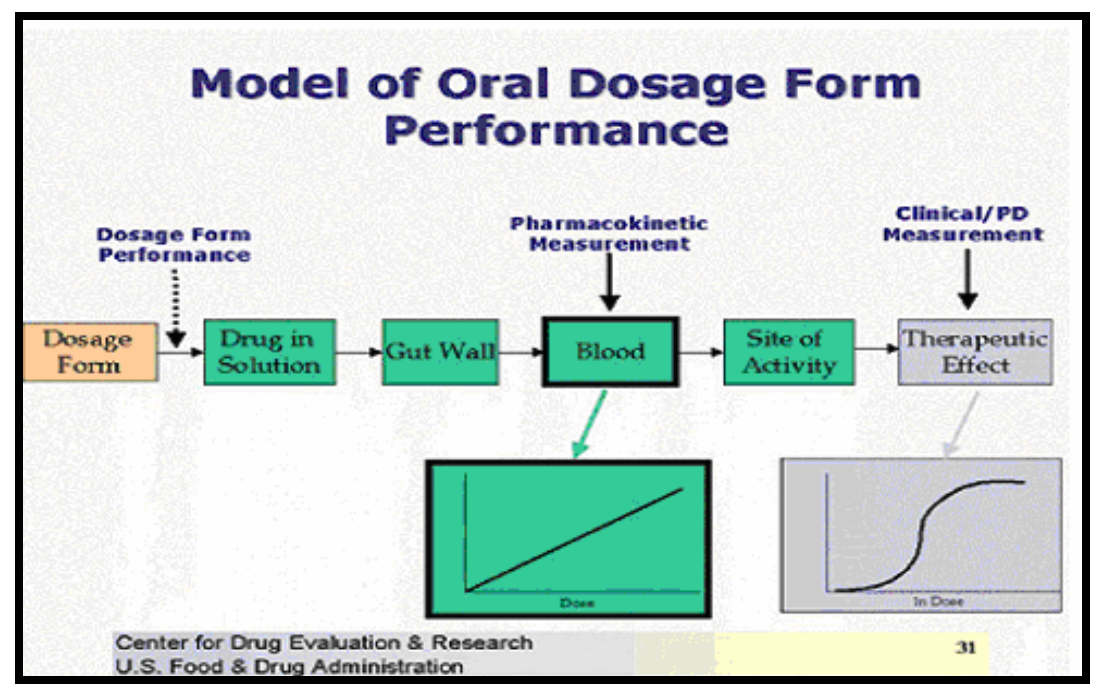

FIGURE 2: Sequence of the fate of drug after oral administration ${ }^{8}$

Availability of a drug candidate in solution form at the absorption site is very important factor for the successful development and commercialization of new drugs \& the dosage form in the pharmaceutical industry. No matter how active or potentially active a new molecular entity (NME) is against a particular molecular target, if it does not possess the above said property, it can not be a viable candidate for further develop ment ${ }^{9}$.

Therefore, there is an almost requirement for improving the solubility of the drug candidate. A wide range of different technologies have been evolved in the recent past. A number of techniques of diverse nature are being presently utilized for enhancing the solubility of drugs.

\section{SOLID DISPERS IONS}

In 1961, Sekiguchi and Obi first proposed the utilization of solid dispersions to increase the dissolution and oral absorption of poorly water-soluble drugs. They proposed the formation of a eutectic mixture of a poorly watersoluble drug with a physiologically inert, easily soluble C 2011, JDDT. All Rights Reserved carrier (Chiou and Riegelman, 1969). In 1971 Chiou and Riegelman defined solid dispersion as "the dispersion of one or more active ingredients in an inert carrier matrix at solid-state prepared by the melting (fusion), solvent or melting-solvent method". The solid dispersions may also be called solid-state dispersions, as first used by Mayersohn and Gibaldi (1966). Corrigan defined the term as "product formed by converting a fluid drug-carrier combination to the solid state" (Corrigan 1985). In a recent review work by Dhirendra et al. adopted the definition given by Chiou and Riegelman "a group of solid products consisting of at least two different components, generally a hydrophilic matrix and a hydrophobic drug". The matrix can be either crystalline or amorphous. The drug can be dispersed molecularly, in amorphous particles (clusters) or in crystalline particles" (Dhirendra et al., 2009). But if the drug is converted to amorphous form and forms one phase system with polymer, it can be classified as a solid solution, whereas if the drug exists as microcrystalline dispersion, i.e., forms two-phase system, it is generally 
referred to as a solid dispersion (Go ldberg et al., 1965; Sekiguchi and Obi 1961; Chokshi et al., 2007) ${ }^{10}$.

Solid dispersion is a well-known approach for improvement of the dissolution rate and bioavailability of drugs that are poorly water soluble. To achieve faster dissolution rate of poorly water-soluble drug, the drug is dispersed at molecular level in a rapidly water-soluble inert carrier to form a solid dispersion. Successful dispersion of the drug in the carrier, at molecular level, leads to formation of homogeneous phase of the solid dispersion. When such a product comes in contact with gastric fluid, then the water-soluble carrier rapidly dissolves leading to immediate release of the drug at the desired molecular level to cause dissolution with consequent improvement of bioavailability. Solid dispersions have been extensively studied for improvement of dissolution rate and numerous techniques of diverse nature have been developed for preparation of the same ${ }^{11}$.

Because of the simplicity of manufacturing and scale up processes, the popularity of the solid dispersion systems to solve difficult bioavailability issues with respect to poorly water-soluble drugs will grow rapidly. Because the dosage form can be developed and prepared by using small amounts of drugs substances in early stages of the drug development process, the system might have an advantage over such other commonly used bioavailability enhancement techniques as micronization of drugs and soft gelatin encapsulation. Single or combination of carriers may also be essential for development of solid dispersion $^{12}$.

The drug in the solid dispersion may present as crystalline particles, amorphous particles or molecularly dispersed; as shown in the figure 3 :

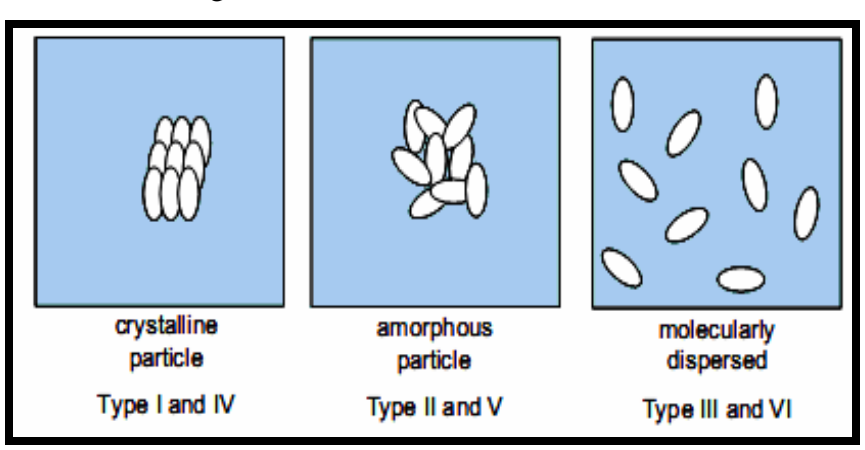

Figure 3: Forms of the drug in solid dispersion ${ }^{13}$

\section{IDEAL DRUG CANDIDATES FOR SOLID DISPERS ION}

In the Biopharmaceutical Classification System (BCS) class II drugs are those with low aqueous solubility and high me mbrane permeability and therefore solid dispersion technologies are particularly promising for improving the oral absorption and bioavailability of BCS Class II drugs . According to the BCS, drug substances can be classified as belonging to one of four classes, Ahmad Zaheer et al $(2011)^{14}$.

Class I: High permeability and solubility.

Formulation independent: - The bioavailability of class I compounds is determined only by delivery of the drug solution to the intestine.
Class II: high permeability but low solubility

Formulation dependent: - The bioavailability of class II compounds is limited by drug solubility/dis solution.

Examples: Vals artan, Nimesulide, Loratadine, Aceclo fenac etc.

\section{Class III: low permeability but high solubility}

Dependent on barrier properties : - The bioavailability of class III compounds is limited by intestinal permeability.

Examples: Gabapentine, Topiramate, Atropine etc.

\section{Class IV: low permeability and low solubility}

Formulation and barrier properties dependent: - The bioavailability of class IV compounds is limited both by solubility / dis solution and intestinal permeability.

Examples: Hydrochlorthiazide, Fu rosemide, Melo xicam etc.

Among the four classes class II drugs shows poor solubility and high permeability. It is obvious that for class II drugs the low ability to dissolve is a more important limitation to their overall rate and extent of absorption then their ability to permeate through the membrane. Therefore, the formulation work for class II compounds should focus on the enhancement of aqueous solubility or dissolution rate ${ }^{15}$.

\section{CLASSIFICATION OF SOLID DISPERSION}

(1) Simple Eutectic Mixtures: A simple eutectic mixture consists of two compounds which are completely miscible in the liquid state but only to a very limited extent in the solid state ${ }^{16}$.

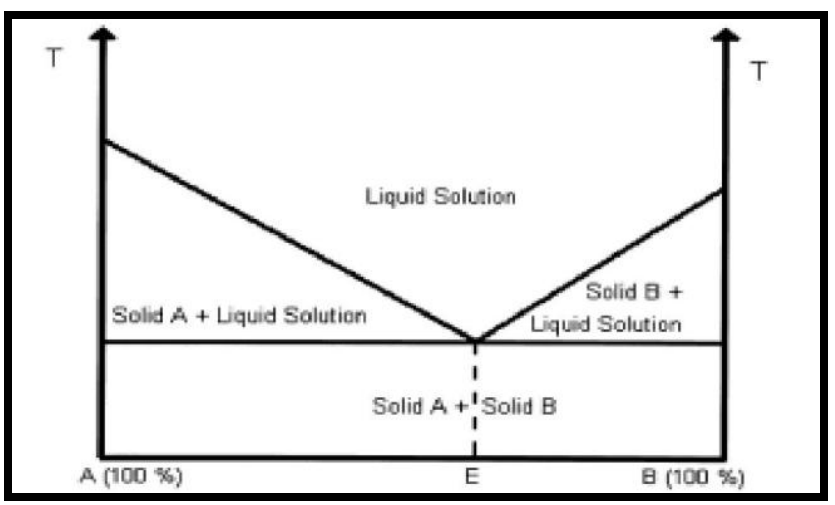

Figure 4: Phase diagram for a Eutectic mixture ${ }^{16}$

(2) Amorphous Precipitations in a Crystalline Carrier: This type of solid dispersion is distinguished from a simple eutectic mixtu re by the fact that the drug is precipitated out in an amorphous form. In a simple eutectic mixture, the drug is precipitated out in a crystalline form. It is postulated that a drug with a propensity to super cooling has more tendency to solidify as an amorphous form in the presence of a carrier ${ }^{17}$.

(3) Solid solutions: Solid solutions of a poorly water soluble drug dissolved in a carrier with relatively good aqueous solubility are of particular interest as a means of improving oral bioavailability. In the case of solid solutions, the drug's particle size has been reduced to its absolute minimum viz. the molecular dimensions and the 
dissolution rate are determined by the dissolution rate of the carrier $^{17}$.

3.1) Continuous solid solutions - In a continuous solid solution, the components are miscible in all proportions. Theoretically, this means that the bonding strength between the two components is stronger than the bonding strength between the molecules of each of the individual components. Solid solutions of this type have not been reported in the pharmaceutical literature to date ${ }^{16}$.

3.2) Discontinuous solid solutions- In the case of discontinuous solid solutions, the solubility of each of the components in the other component is limited. The phase diagram in Fig. 5 shows the regions of true solid solutions. In these regions, one of the solid components is completely dis solved in the other solid component ${ }^{17}$.

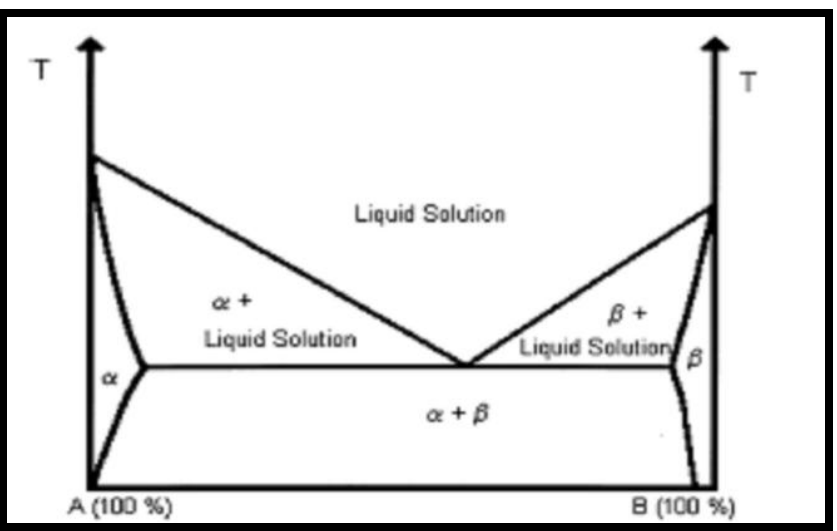

Figure 5: Phase diagram for a discontinuous solid solution $^{17}$

3.3) Substitutional crystalline solid solutions - Clas sical solid solutions have a crystalline structure, in which the solute molecules can either substitute for solvent molecules in the crystal lattice or fit into the interstices between the solvent molecules. Substitution is only possible when the size of the solute molecules differs by less than $15 \%$ or so from that of the solvent molecules ${ }^{17}$.

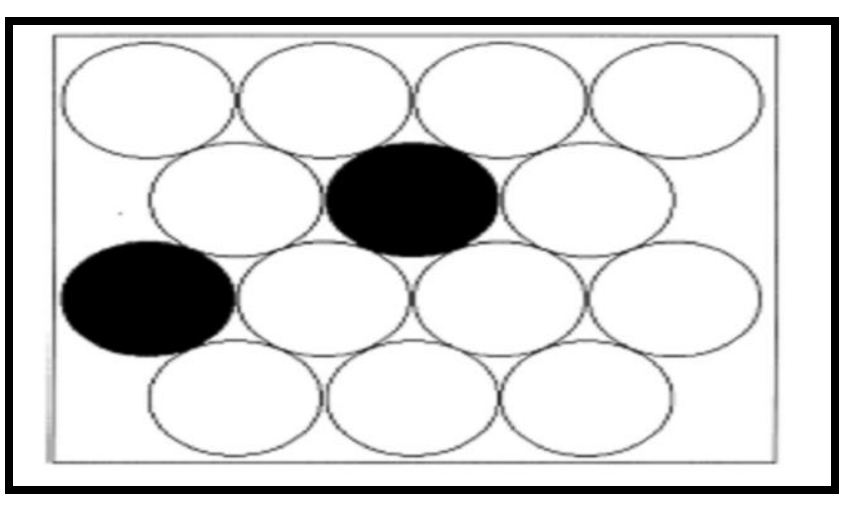

Figure 6: Substitutional crystalline solid solution ${ }^{17}$

3.4) Interstitial crystalline solid solutions: In interstitial solid solutions, the dissolved molecules occupy the interstitial spaces between the solvent molecules in the crystal lattice. In the case of interstitial crystalline solid solutions, the solute molecules should have a molecular diameter that is no greater than 0.59 of the solvent molecule's molecular diameter.

3.5) Amorphous solid solutions: In an amorphous solid solution, the solute molecules are dispersed molecularly but irregularly within the amorphous solvent. Polymer (c) 2011, JDDT. All Rights Reserved carriers (viz. PVP, PEG, cellulose derivatives etc) are particularly likely to form amorphous`solid solutions as the polymer itself is often present in the form of an amorphous polymer chain network. In addition, the solute molecules may serve to plasticize the poly mer, leading to a reduction in its glass transition temperature ${ }^{16}$.

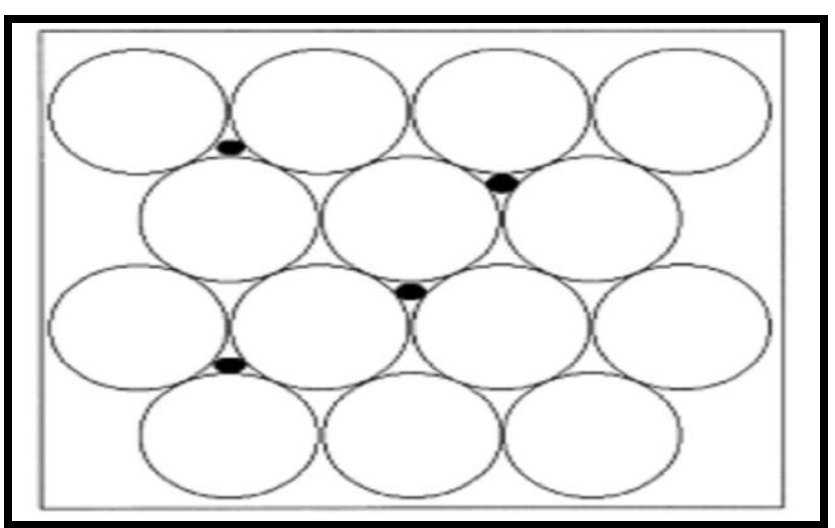

Figure 7: Interstitial crystalline solid solution ${ }^{16}$

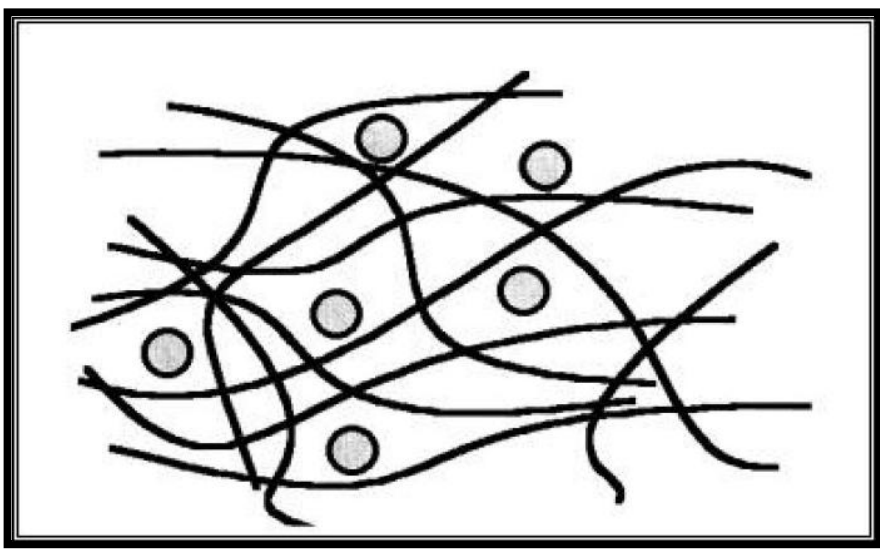

Figure 8: Amor phous solid solution ${ }^{16}$

(4) Glass solution and Glass suspension: A 'Glass solution' is a homogenous, glassy system in which a solute dissolves in the glassy solvent, where as 'Glass suspension' refers to a mixture in which precipitated particles are suspended in a glassy solvent. The glassy state is characterized by transparency and brittleness below the glass transition temperature. Glasses do not have sharp melting points. Instead, they soften progressively on heating. The lattice energy, which represents a barrier to rapid dissolution, is much lower in glass solutions than in solid solutions ${ }^{18}$.

(5) Compound or Complex Formation: Complex formation (soluble and insoluble) can enhance or reduce the dissolution of drugs e.g. Quinidine-Phenobarbitone system increases dissolution whereas PEG-Phenobarbitone system reduces the dissolution. Various types of cyclodextrins are usually used to enhance the solubility of drugs via comple xation ${ }^{19}$.

\section{SELECTION OF CARRIERS USED IN SOLID DISPERS ION}

The selection of the carrier has the influence on the dissolution characteristics of the dispersed drug since the dissolution rate of one component from the surface is affected by the other component in a multi component mixture. Therefore a water soluble carrier results in a faster release of the drug from the matrix. A poorly soluble

CODEN (USA): JDDTAO 
or insoluble carrier leads to slower release of a drug from the matrix. The carriers to be used should have the following characteristics: ${ }^{20}$

1. Readily soluble in water and in gastrointestinal fluids

2. Physiologically inert

3. Melting point not much higher than that of drug

4. Thermal stability at melting temperature

5. Low vapour pressure

6. High molecular weight

7. They should be non toxic

\section{CARRIERS IN SOLID DISPERS ION}

Polyethylene glycol: Polyethylene glycols (PEGs) are polymers of ethylene oxide, with a molecular weight (MW) usually falling in the range $200 \pm 3,00,000$. The ir solubility in water is generally good, but decreases with MW. A particular advantage of PEGs for the formation of solid dispersions is that they also have good solubility in many organic solvents. The melting point of the PEGs of interest lies under $65^{\circ} \mathrm{C}$ in every case (e.g. the m.p. of PEG 1000 is $30-40^{\circ} \mathrm{C}$, the m.p. of PEG 4000 is $50-58^{\circ} \mathrm{C}$ and the m.p. of PEG 20,000 is $\left.60-63^{\circ} \mathrm{C}\right)^{21}$. These relatively low melting points are advantageous for the manufacture of solid dispersions by the melting method. PEGs of MW 4000-6000 are the most frequently used for the manufacture of solid dispersions, because in this MW range the water solubility is still very high, but hygroscopy is not a problem and the melting points are already over $50^{\circ} \mathrm{C}$. If a PEG with too low a MW is used, this can lead to a product with a sticky consistency that is difficult to formulate into a pharmaceutically acceptable product ${ }^{22}$.

Poly vinyl pyrrolidone: Polymerization of vinylpyrrolidone leads to polyvinylpyrrolidone (PVP) of molecular weights ranging from 2500 to $3,000,000$. These can be classified according to the $\mathrm{K}$ value. Due to their good solubility in a wide variety of organic solvents, they are particularly suitable for the preparation of solid dispersions by the solvent method. PVPs have good water solubility and can improve the wettability of the dispersed compound in many cases. The chain length of the PVP has a very significant influence on the dissolution rate of the dispersed drug from the solid dispersion. The aqueous solubility of the PVPs becomes poorer and viscosity lowers with increasing chain length ${ }^{23}$.

Urea: Urea is the end product of human protein metabolism, has a light diuretic effect and is regarded as non-toxic. Its solubility in water is greater than 1 in 1 and it also exhibits good solubility in many common organic solvents. In one of the first bioavailability studies of solid dispersions, it was shown that sulphathiazole was better absorbed in rabbits when given as a eutectic with urea ${ }^{24}$.

Sugars: Although sugars and related compounds are highly water soluble but few sugars have toxicity issues, they are less suitable than other carriers for the manufacture of solid dispersions. Lactose is useful as a carrier for the production of solid dispersions of drugs prepared by melting and rapid cooling showed marked increase in dissolution rate. Chitosan has also been used as a carrier in solid dispersions. Mannitol can be employed in some cases to prepare dispersions by the hot melt $\operatorname{method}^{25}$.

Emulsifiers: The release behaviour of many drugs can also be improved through the use of emulsifying agents. Two mechanisms are possible here: improvement of wetting characteristics and solubilization of the drug. Owing to their potential toxicity problems, such as damage to mucosal surfaces, they are used in combination with another carrier. Surfactants are suitable carriers for low dose and very low water soluble drugs ${ }^{26}$. Poloxamers are nonionic triblock copolymers. Because of their amphiphilic structure, the polymers have surfactant properties that make them useful in industrial applications. The recently used surface-active carrier is Gelucire ${ }^{\circledR}$ 44/14 and other grades of Gelucire®. Bile salts and their derivatives are natural surfactants; they enhance the wetting and solubility of many lipophilic substances, leading to an increase in dissolution rate.

Polyacrylates and polymethacrylates: Polyacrylates and polymethacrylates are glassy substances that are produced by polymerization of acrylic and methacrylic acid, and derivatives of these polymers such as esters amides and nitriles. In pharmaceuticals they are mostly used in coatings to modify the release of the drug from the dosage form. Commonly they are referred by the trade name Eudragit $^{25}$.

Cellulose derivative: Celluloses are naturally occurring polysaccharides that are ubiquitous in the plant kingdom. They consist of high molecular weight unbranched chains, in which the saccharide units are linked by $\beta-1$, 4glycoside bonds. By appropriate alkylation, the cellulose can be derivatized to form methyl- (MC), hydroxypropyl (HPC), hydroxypropylmethyl (HPMC) and many other semi-synthetic types of cellulose. A further possibility for derivatization is the esterification of the cellulose to form compounds such as cellulose acetate phthalate (CAP) and hydroxypropylmethylcellulose phthalate (HPMCP) ${ }^{25}$.

\section{ADVANTAGES OF SOLID DISPERS IONS}

1. Particles with reduced particle size and increased dissolution rate: Solid dispersions represent the last state on particle size reduction and after carrier dis solution the drug is molecularly dispersed in the dissolution medium. Solid dispersions apply this principle to drug release by creating a mixture of a poorly water soluble drug and highly soluble carriers. Due to this a high surface area is formed resulting in an increased dissolution rate and improved bioavailability ${ }^{27}$.

2. Particles with improved wettability: A strong contribution to the enhancement of drug solubility is related to the drug wettability improvement verified in solid dispersions. It was observed that even carriers without any surface activity, such as urea improved drug wettability. Carriers with surface activity such as cholic acid and bile salts when used can significantly increase the wettability property of drug. Moreover, carriers can influence the drug dissolution profile by direct dissolution or co-solvent effects.

3. Particles with higher porosity: Particles in solid dispersions have been found to have a higher degree of porosity. The increase in porosity depends on the carrier properties, for instance solid dispersions containing linear 
polymers produce larger and more porous particles than those containing reticular polymers and so result in a higher dissolution rate. The increased porosity of solid dispersion particles also hastens the drug release profile ${ }^{27}$. higher solubility. The enhancement of drug release can usually be achieved using the drug in its amorphous state, because no energy is required to break up the crystal lattice during the dissolution process ${ }^{27}$.

4. Drugs in amorphous state: Poorly water soluble crystalline drugs, when in the amo rphous state tend to have

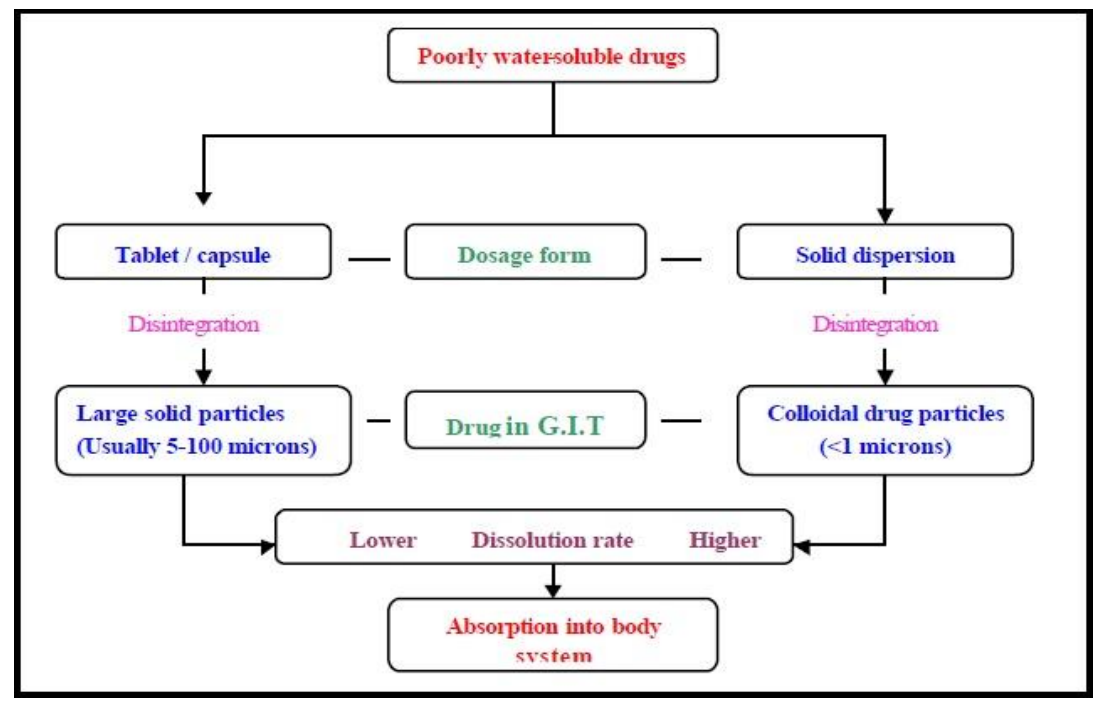

Figure 9: Advantages of a solid dis persion formulation, as compared to conventional capsule or tablet ${ }^{28}$.

\section{DISADVANTAGES OF SOLID DISPERS IONS}

1. Laborious and expensive methods of preparation,

2. Stability of the drug and vehicle.

3. Due to tackiness in some solid dispersion, it sometimes leads to handling problem.

4. Reproducibility of physicochemical characteristics.

5. Difficulty in incorporating into formulation of dosage forms.

6. Scale-up of manufacturing process.

\section{METHODS OF PREPARATION}

Fusion method: Accurately weighed drug and carrier are mixed using glass mortar and pestle. The mixture is heated at or above the melting point of all the components to achieve a homogenous dispersion. It is then cooled to obtain a congealed mass. It is pulverized and sieved. Ex. albendazole and urea solid dispersion was prepared this $\operatorname{method}^{29}$.

Advantages: The main advantage of direct melting method is its simplicity and economy. In addition melting under vacuum or blanket of an inert gas such as nitrogen may be employed to prevent oxidation of drug or carrier.

Disadvantages: Firstly, a major disadvantage is that the method can only be applied when drug and matrix are compatible and when they mix well at the heating temperature. Secondly, a problem can arise during cooling when the drug-matrix miscibility changes. In this case phase separation can occur. Thirdly, degradation of the drug and or matrix can occur during heating to temperatures necessary to fuse matrix and drug.

Solvent method: Drug and carrier both are dissolved in organic solvent. After complete dissolution, the solvent is evaporated. The solid mass is ground, sieved and dried. Ex. Solid dispersion of ofloxacin with polyethylene glycol was prepared by solvent evaporation method ${ }^{29}$.

Advantages: The main advantage of the solvent method is that thermal decomposition of drugs or carriers can be prevented because of the low temperature required for evaporation of organic solvents.

Disadvantages: The disadvantages include the higher cost of preparation, the difficulty in completely removing liquid solvent.

Supercritical fluid methods: Supercritical fluid methods are mostly applied with carbon dioxide, which is used as either a solvent for drug and matrix or as an anti-solvent. When supercritical $\mathrm{CO} 2$ is used as solvent, matrix and drug are dissolved and sprayed through a nozzle, into an expansion vessel with lower pressure and particles are immediately formed. The adiabatic expansion of the mixture results in rapid cooling. This technique does not require the use of organic solvents and since $\mathrm{CO} 2$ is considered environmentally friendly, this technique is referred to as 'solvent free'. The technique is known as Rapid Expansion of Supercritical Solution ${ }^{29}$.

Advantages: The supercritical anti-solvent rapidly penetrates into the droplets, in which drug and matrix become supersaturated, crystallize and form particles. The general term for this process is precipitation with compressed anti- oven.

Disadvantages: Usually organic solvents like dichloromethane or methanol have to be applied to dissolve both drug and matrix which are more in cost.

Melting solvent method: In this method drug is first dissolved in a suitable liquid solvent Solution is then incorporated directly into the melt of polyethylene glycol obtainable below 70C, without removing the liquid 
solvent. It has been shown that $5-10 \%(\mathrm{w} / \mathrm{w})$ of liquid compound could be incorporated into polyethylene glycol 6000 without significant loss of its solid property ${ }^{30}$.

Advantages: In this method that thermal decomposition of drugs or carriers can be prevented because of the low temperature required for evaporation of organic solvents.

Disadvantages: As the practical point of view, the melting-solvent method is limited to drugs with a low therapeutic dose, e.g., below 50mg.

Melt agglomeration process: This technique has been used to prepare solid dispersion where the binder acts as a carrier. Binder (carrier), drug and excipients are heated to temperature above the melting point of the binder (melt-in procedure) or by spraying a dispersion of drug in molten binder on the heated excipient (spray-on procedure) by using a high shear mixer. The rotary process or might be preferable to the high melt agglomeration because it is easier to control the temperature and higher binder content can be incorporated in the agglomerates. Larger particles result in densification of agglomerates while fine particle cause complete adhesion to the mass to bowl shortly after melting attributed to distribution and coalescence of the fine particles ${ }^{30}$.

Spray drying: It is a method which is initiated by atomizing suspensions or solutions into fine droplets followed by a drying process, resulting in solid particles. The process allows production of fine, dust free powder as well as agglomerated one to precise specifications. The operating conditions and dryer design depends upon the drying characteristics of the product and require powder specifications. The spray drying technique is a useful method to obtain spherical particle and narrow distribution $^{31}$.

The use of surfactant: Surfactant reduces hydrophobicity of drug by reducing interfacial or surface tension and because of this unique property surfactants have attracted the attention of investigators for preparation of solid dispersions. Recently a new class of surfactant known as Gelucires are introduced which identify by melting points and HLB values. Gelucire is widely used in the formulation of solid dispersions. Poloxamer represent another class of surfactants. Polys orbate 80 a surfactant results in improvement of dissolution and bioavailability of poorly water soluble drug attributed to the solubilisation effect of surface active agent ${ }^{32}$.

\section{Alternative strategies}

\section{Spraying on sugar beads using a fluidized bed coating system:}

The approach involves a fluidized bed coating system, wherein a drug-carrier solution is sprayed onto the granular surface of excipients or sugar spheres to produce granules ready for tabletting or drug coated pellets for encapsulation in one step. The method has been applied for both controlled and immed iate release solid dis persions ${ }^{33}$.

Itraconazole (Sporanox oral capsules, Janssen Pharmaceutica, Titusville, NJ) coated on sugar sphere, is made by layering onto sugar beads a solution of drug and hydroxypropylmethylcellulose (HPMC) in an organic solvent of dichloromethane and ethanol. A solid solution of drug in HPMC is produced upon coating (cosolvent evaporation) and controlled drying of coated beads in a closed Wurster process. As this thin film dissolves in water or gastric fluid, the molecularly dispersed itraconazole is released at supersaturated concentration. HPMC acts as a stabilizer to inhibit recrystallization of the itraconazole. The supersaturated solutions of itraconazole are sufficiently stable to allow for absorption and distribution.

Hot melt extrusion: It has been reported that melt extrusion of miscible components results in amorphous solid solution formation, whereas extrusion of an immiscible component results to amorphous drug dispersed in crystalline excipient ${ }^{34}$. The process has been useful in the preparation of solid dispersions in a single step. Hot-melt extrusion method is used in the preparation of various dosage forms in the pharmaceutical industry such as preparation of sustained-release pellets. The advantages of hot-melt extrusion include lower temperature and shorter residence time of the drug carrier mix ( $<2$ minutes), absence of organic solvents, continuous operation possibility, minimum product wastage, good control of operating parameters, and possibility to scale up. The disadvantages are few and mainly relate to negative effects of shear force.

Direct capsule filling: The filling of semisolid materials into hard gelatin capsules as melts, which solidify at room temperature, was first done in 1978. Laboratory-scale semiautomatic equipment and large-scale manufacturing equipment for direct capsule filling are commercially available. Direct filling of hard gelatin capsules with the liquid melt of solid dispersions avoids grinding-induced changes in the crystallinity of the drug. The filling of hard gelatin capsules has been feasible in molten dispersions of triamterene-PEG 1500 using a Zanasi LZ 64 capsulefilling machine (Zanasi Co, Bologna, Italy). This molten dispersion forms a solid plug inside the capsule on cooling to room temperature, reducing crosscontamination and operator exposure in a dust-free environment better fill weight and content uniformity was obtained than with the powder-fill technique. However, PEG was not a suitable carrier for the direct capsule-filling method as the watersoluble carrier dissolved more rapidly than the drug, resulting in drug-rich layers formed over the surface of dissolving plugs, which prevented further dissolution of the drug ${ }^{35}$. A surfactant must be mixed with the carrier to avoid formation of a drug-rich surface layer (eg, polysorbate 80 with PEG).

Electrostatic spinning method: The electrostatic spinning method technology used in the polymer industry combines solid solution/dispersion technology with nanotechnology. This technology is now applied in the pharmaceutical field. In this process, a liquid stream of a drug/polymer solution is subjected to a potential between 5 and $30 \mathrm{kV}$. When electrical forces overcome the surface tension of the drug/polymer solution at the air interface, fibers of submicron diameters are formed. Water-soluble polymers would be useful in the formulation of immediate release dosage forms, and water-insoluble (both biodegradable and nonbiodegradable) polymers are useful in controllable dissolution properties. Fabrics generated by water-soluble carriers could be used in oral dosage formu lations by direct incorporation of the materials into a capsule. 
Surface active carriers: A surface-active carrier may be preferable in almost all cases for the solid dispersion of poorly water-soluble drugs. In addition to their use as excipients to improve the physical and chemical characteristics of the formulation, surface active carriers may be included to improve the efficacy of the product. The properties of surfactant are such that they can alter the thermodynamic activity, solubility, diffusion, disintegration and dissolution rate of a drug. Each of these parameters influences the rate and extent of drug absorption. Surface active carriers can exert direct effects on biological membranes thus altering drug transport across the membrane ${ }^{36}$. The surface-active and selfemulsifying carriers for solid dispersion of poorly watersoluble drugs have been of great interest in recent years. Two of the important surface-active carriers are Gelucire 44/14 and Vitamin E R-alpha-tocopheryl polyethylene glycol 1000 succinate (TPGS).

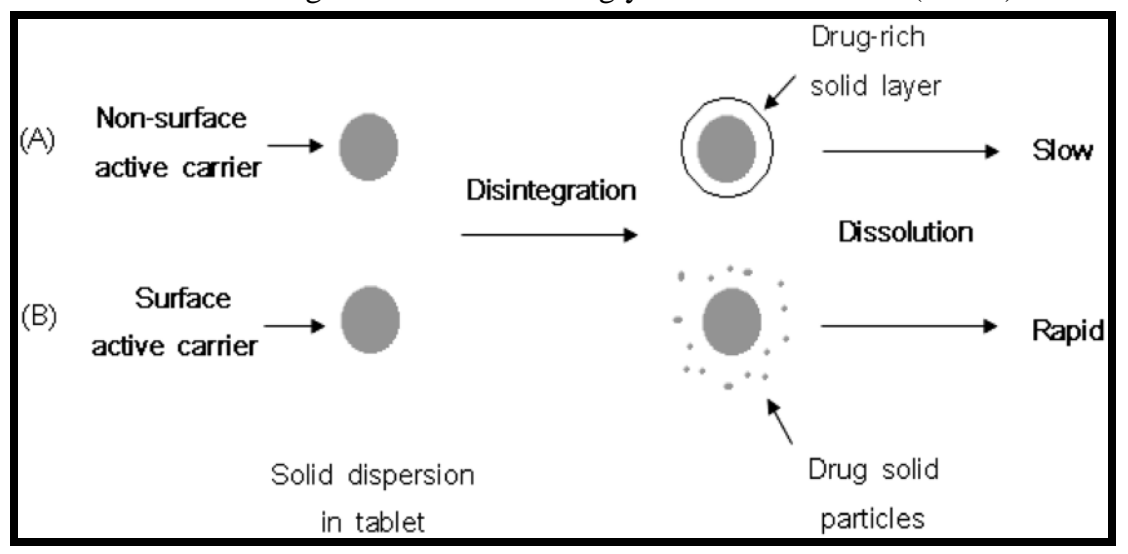

Figure 10: Relative dissolution rate of solid dis persions containing surface acti ve carrier, compared to non-surface active carrier ${ }^{37}$

\section{CHARACTERIZATION OF SOLID DISPERS ION}

A.Detection of crystallinity in solid dispersions: Several different molecular structures of the drug in the matrix can be encountered in solid dispersions. Many attempts have been made to investigate the molecular arrangement in solid dispersions. Many techniques are available which detect the amount of crystalline material in the dispersion.

Currently, the following techniques are available to de tect the crystallinity:

1. Powder X-ray diffraction: It can be used to qualitatively detect material with long range order. Sharper diffraction peaks indicate more crystalline material $1^{38}$.

2. Infrared spectroscopy (IR): It can be used to detect the variation in the energy distribution of interactions between drug and matrix. Sharp vibrational bands indicate crystallinity. Fourier Transformed Infrared Spectroscopy was used to accurately detect crystallinities ranging from 1 to $99 \%$ in pure material $1^{39}$.

3. Isothermal Microcalorimetry: It measures the crystallization energy of amorphous material that is heated above its glass transition temperature $(\mathrm{Tg})^{39}$.

4. Dissolution Calorimetry: It measures the energy of dissolution, which is dependent on the crystallinity of the sample; usually dissolution of crystalline material is endothermic, whereas dissolution of amorphous material is exothermic ${ }^{40}$.

5. Macroscopic techniques can be indicative for the degree of crystallinity. Density measurements and Dynamic Mechanical Analysis (DMA) determine the modulus of elasticity and viscosity and thus affected by the degree of crystallinity.

6. A technique used to detect the amount of crystalline material is Differential Scanning

(c) 2011, JDDT. All Rights Reserved
Calorime try (DSC). In DSC, samples are heated with a constant heating rate and the amount of energy necessary for that is detected. With DSC the temperatures at which thermal events occur can be detected. Also the melting and crystallization energy can be quantified. The melting energy can be used to detect the amount of crystalline material.

B.Detection of molecular structure in amor phous solid dis persions

The properties of a solid dispersion are highly affected by the uniformity of the distribution of the drug in the matrix. The following techniques are used:

1. Confocal Raman Spectroscopy was used to measure the homogeneity of the solid mixture of ibuprofen in PVP. It was described that a standard deviation in drug content smaller than $10 \%$ was indicative of homogeneous distribution ${ }^{41}$.

2. Using IR or FTIR, the extent of interactions between drug and matrix can be measured. The interactions are indicative for the mode of incorporation of the drug, because separately dispersed drug molecules will have more drug-matrix interactions than when the drug is present in amorphous clusters ${ }^{42}$.

3. Temperature Modulated Differential Scanning Calorimetry (TMDSC) can be used to assess the degree of mixing of an incorporated drug. Due to the modulation reversible and irreversible events can be separated. This technique can be used to assess the amount of molecularly dispersed drug and from that the fraction of drug that is dispersed as separate molecules is calculated ${ }^{43}$.

\section{MARKETED PRODUCTS}

Commercial products based on solid solution / dispersion are: 
1. Gris-PEG, a griseofulvin-PEG fusion method solid dispersion, was manufactured initially by Dorsey / Sandoz and reached the market in the mid1970s.Gris-PEG was developed as tablet product, and this led to two USP monographs for griseofulvin tablets. The solid dispersion form is referred to as ultramicrosize griseofulvin tablets USP and offers improved bioavailability and two- thirds reduced dosage compared to griseofulvin tablets USP. The griseofulvin tablets USP are manufactured from micronized drug substance using a conventional tabletting approach. Griseofulvin solid dispersion tablets are currently marketed by a number of manufacturers and contain corn starch, lactose, magnesium stearate, PEG, and sodium lauryl sulfate as inactive ingredients ${ }^{29}$.

2. Cesamet, a nabilone-PVP solvent method solid dispersion manufactured by Eli Lilly and Co. has been marketed internationally since 1982. Eli Lilly discontinued marketing Cesamet contains PVP and corn starch as inactive ingredients and is presented as a capsule product ${ }^{44}$.

3. Solid dispersion formulation of Troglitazone (Rezulin) is marketed by Parke-Dav is ${ }^{45}$.

4. Solid solutions of lopinavir and ritonavir in polyvinylpyrrolidone-vinyl acetate copolymer successfully enabled a reformulation of "Kaletra" ( Abbott Laboratories, Abbott Park, IL). In addition to reducing the dosage burden from six softgel capsules to four tablets, tablets made with the solid solutions eliminate the need for refrigeration ${ }^{29}$.

5. "Sporanox" (Janssen Pharmaceutica, Titusville, NJ) is a solid dispersion of itraconazole in hypromellose that has been layered onto sugar spheres ${ }^{29}$.

6. The most recently approved product is the nonnucleoside reverse transcriptase inhibitor "Intelence" (Tibotec, Yardley, PA), an amorphous, spray-dried solid dispersion of etravirine, hypromellose, and microcrystalline cellu los $\mathrm{e}^{46}$.

\section{APPLICATION OF SOLID DISPERS ION IN PHARMA INDUS TRIES}

1. Increases oral bioavailability of poorly water-soluble drugs

2. Solid state suitable for oral delivery

3. No change in chemical properties of the drug

4. Relatively simple processing techniques

5. Uses conventional equip ment

6. Increases dissolution due to metastable solid state

7. To obtain a homogeneous distribution of a small amount of drug in solid state

8. To stabilize the unstable drug.

9. To dispense liquid (up to $10 \%$ ) or gaseous compounds in a solid dosage.

10. To formu late a fast release primary dose in a sustained released dosage form.
11. To formulate sustained release regimen of soluble drugs by using poorly soluble or insoluble carriers.

12. To reduce pre systemic inactivation of drugs like morphine and progesterone.

\section{FUTUR E POTENTIAL OF SOLID DISPERS ION}

The solubility of drugs in aqueous media is a key factor highly influencing their dissolution rate and bioavailability following oral administration resulting in low bioavailability. Solubility enhancement of these drugs remains one of the most challenging aspects of drug development. A variety of devices have been developed over the years to enhance the drug solubility and dissolution of the drugs. The solid dispersion method is one of the effective approaches to achieve the goal of solubility enhancement of poorly water-soluble drugs. Various techniques, described in this review, are successfully used for the preparation of $\mathrm{SD}(\mathrm{s})$ in the bench and lab scale and can be used at industrial scale also. Solid dispersions came into limelight in pharmaceutical development due to the increasing number of drug candidates which are poorly soluble and the substantial improvements in the manufacturing methods for solid dispersions that have been made in the last few years. Although there are some hurdles like scale up and manufacturing cost to overcome, there lies a great promise that solid dispersion technology will hasten the drug release profile of poorly water soluble drugs, Ahuja et al (2007).

Despite many advantages of solid dispersion, issues related to preparation, reproducibility, formulation, scale up, and stability limited its use in commercial dosage forms for poorly water-soluble drugs. Successful developments of solid dispersion systems for preclinical, clinical and commercial use have been feasible in recent years due to the availability of surface-active and self-emulsifying carriers with relatively low melting points. The preparation of dosage forms involves the dissolving of drugs in melted carriers and the filling of the hot solutions into gelatin capsules. Because of the simplicity of manufacturing and scale up processes, the physicochemical properties and as expected to change significantly during the scale up. For this reason, the popularity of the solid dispersion systems to solve difficult bioavailability issues with respect to poorly water-soluble drugs will grow rapidly.

\section{CONCLUSION}

As poorly water-soluble compounds become more prevalent in the pharmaceutical markets, there are increased attempts for enhancing oral bioavailability of these drug candidates. Solid dispersions are one of the most promising strategies to solve this problem. Experience with solid dispersions over the last 20-30 years indicates that this is a very fruitful approach to improving the release rate and oral bioavailability of poorly water soluble drugs and the availability of a wide variety of polymers that are themselves poorly soluble or which swell under aqueous conditions suggests that solid dispersions have tremendous potential in the area of controlled release dosage forms. Because of solubility problem of many drugs the bio availability of them gets affected and hence solubility enhancement becomes necessary. Solid dispersion technology is one of the 
possible modes that increase the solubility of poorly soluble drugs. Successful development of solid dispersion system for preclinical, clinical and commercial use has been feasible in recent years due to the availability of surface active carriers and self emulsifying carriers.

\section{REFERENCES}

1. Oral delivery of poorly soluble drugs, from pharmapedia - the free pharmaceutical encyclopedia, http://www.pharmpedia.com.

2. Stella V, Borchardt R, Hageman M, Oliyai R, Maag H and Tilley J. Biotechnology : Pharmaceutical Aspects, Edition 2007, 5(2), Publisher- Springer New York, pg. no. 157-215.

3. Lindenberg M, Kopp S, Dressman J, Classification of orally administered drugs on the WHO model list of essential medicines according to biopharmaceutical classification system, European Journal Of Pharmaceutics \& Biopharmaceutics, 2004, 58( 2), 265 278.

4. Chaudhari PD, Current trend in solid dispersion techniques, www.pharmainfo.net, 2006, 2-12.

5. Kommu A, Chebrolu J B, Laksmaiah P, Chandu B R, Techniques to improve the absorption of poorly soluble drugs, IJRPC, 2012, 2(2), 335-540.

6. Milo Gibaldi. Bio pharmaceutics and clinical pharmacokinetics, Gastro intestinal absorption- physicochemical consideration, 4th Edition, 1991, 46, 75-78.

7. http://www.drugdeliverytech.com/cgi-bin/articles.cgi?idArticle=262 (accessed February 20, 2010).

8. Gupta V, Madan AK, Agarwal R, Rapidly soluble tablets through amalgamation of cube sugar and solid dispersion technologies, IJPFR, 2011, 1(2), 1-13.

9. Martin A. Physical pharmacy, Pennsylvania: Lea \& Febiger, 1993, 213.

10. Saffoon N, Uddin R, Huda HN and Kumar BS, Enhancement of oral bioavailability and solid dispersion: a review, JAPS, 2011, 1(7), 13 20.

11. Goldberg AH, Galbaldi M, Kanig KL, Increasing dissolution rates and gastrointestinal absorption of drugs via solid solutions and eut ectic mixtures III, J. Pharm. Sci., 1966, 55, 487-492.

12. Christensen KL, Design of Redispersible Dry Emulsion: A Potential Oral Drug Delivery System, PhD Thesis 2000, 35-45.

13. Dhirendra K, Lewis S, Udupa N and Atin K, Solid dispersion: A Review, Pak. J. Pharm. Sci., 2009, 22(2), 234-246.

14. Zaheer A, Maurya N, Mishra K. S, Khan I, Solubility enhancement of poorly soluble drugs: A review, IJPT, 2011, 3(1), 807-823.

15. Giri KT, Alexander A, Tripathi KD, Physicochemical Classification and Formulation Development of Solid Dispersion of Poorly Water Soluble Drugs: An Updated Review, IJPBA, 2010, 1(4), 309-324.

16. Leuner C, Dressman J, Improving drug solubility for oral delivery using solid dispersions, Eur. J. Pharm, 2000, 47-60.

17. Vadnere MK.. Encyclopedia Of Pharmaceutical Technology, 2nd Ed.., Vol.1, Ed. by Swarbric J., Boylan J.C., Marcel Dekker Inc., New York, 2002, 641-648.

18. Ford JL, The current status of solid dispersions, Pharm. Acta Helv. 61, 1986, 69-88.

19. Chiou WL, Riegelman S, Pharmaceutical application of solid dispersion system, J.Pharm.Sci.60, 1971, 1281-1302.

20. Arunachalam A, Karthikeyan M, Konam K, Prasad P, Sethuraman S, Ashutoshraman S, Solid dispersions: a review, CPR, 2010, 1:82-90.

21. Price JC. Polyethylene glycol. In: Wade A, Weller PJ, Ed. Handbook of Pharmaceutical Excipients, Washington DC/London: American Pharmaceutical Association/The Pharmaceutical Press, 1994, $355-$ 361 .

22. Shah JC, Chen JR, Chow D, Preformulation study of etoposide, increased solubility and dissolution rate by solid-solid dispersions, Int J Pharm, 1995, 103-111.

23. Walking WD. Povidone. In: Wade A, Weller PJ, Ed. Handbook of Pharmaceutical Excipients, Washington DC/London: American Pharmaceutical Association/The Pharmaceutical Press, 1994, 392 399.

24. Sekiguchi K, Obi N, Studies on Absorption of Eutectic Mixture, A comparison of the behaviour of eutect ic mixture of sulfathiazole and of ordinary sulfathiazole in man, Chem Pharm Bull, 1961, 866-872.
ACKNOWLEDGEMENT: Authors are highly acknowledged to Ms Vandana Dhillon from Loyd Institute of Management and Technology, Greater Noida for his valuable suggestions, necessary help, and support and to the Library Department of LIMT, for providing literature facilities for preparation of this review article.

25. Sharma A, Jain CP, Solid dispersion: A promising technique to enhance solubility of poorly water soluble drug, International Journal of Drug Delivery, 2011, 149-170.

26. Shokri J, Azarmi S, Saboury A, Shokri MH, Enhancement of oxazepam dissolution rate using oxazepam-surfactant solid dispersions, Ulum-i Daroei, 2006, 4:35-45.

27. Sharma D, Soni M, Kumar S and Gupta GD, Solubility Enhancement - Eminent Role in Poorly Soluble Drugs, Research Journal Pharm. And Tech., 2009, 2(2), 220-224.

28. Kamalakkannan V, Puratchikody A, Masilamani K, Senthilnathan B, Solubility enhancement of poorly soluble drugs by solid dispersion techniques- A Review, JPR, 2010, 3(9), 2314-2321.

29. Kalyanwat R, Patel S, Solid Dispersion: A method for enhancing drug dissolution, IJDFR, 2010, Vol.1, 1-14

30. Chiou WL and Reigelman S, Pharmaceutical applications of solid dispersion systems, JPharm Sci, 1971, 60:1281-1302.

31. Visser MR, Zijlastra GS, Frijlink HW, Phsicochemical classification and formulation development of solid dispersion of poorly water soluble drugs: an updated review, Int J Pharm, 2010, 1:49-58.

32. Verheyen S, Blaton N, Kinget R, Mooter VD, Mechanism of Increased Dissolution of Diazepam and Temazepam from Polyethylene Glycol 6000 Solid Dispersions, Int J Pharm, 2002, 24:45-58.

33. Ho Ho, Shu HL, Tsai T, Sheu MT, The preparation and characterization of solid dispersions on pellets using a fluidized bed system, Int JPharm, 1996, 13:223-29.

34. Breitenbach J, Melt extrusion: from process to drug delivery technology, Eur J Pharm Biopharm, 2002, 54:107-17.

35. Serajuddin ATM, Sheen PC, Mufson D, Bernstein DF, Augustine MA, Effect of vehicle amphilicity on the dissolution and bioavailability of a poorly water soluble drug from solid dispersions, J Pharm Sci, 1988, 77:414-17.

36. Vansihv SD, Rao MRP, Sonar GS, Gogad VK, Borate SG, Physicochemical characterization and In Vitro Dissolution of Domperidone by Solid Dispersion Technique, Indian J Pharm Educ Res, 2009, 43:86-90.

37. Kim TK, Lee YJ, Lee YM, Song KC, Kim DD, Solid dispersions as a drug delivery system, Journal of Pharmaceutical Investigation, 2011, 41(3), 125-142.

38. Ahmad M, Fattah A, Bhargava HN, Preparation and evaluation of solid dispersions of halofantrine, Int J Pharm, 2002, 23:17-33.

39. Kumar SD, Gupta VB, Purohit S, Solubility improvement using solid dispersion strategy, mechanism and characterization: responsiveness and prospective way outs, IRJP, 2011, 2:55-60.

40. Drooge V, Characterization of the molecular distribution of drugs in glassy solid dispersions at the nano-meter scale using differential scanning calorimetry and gravimetric water vapour sorption techniques, Int J Pharm, 2006, 31:220-29.

41. Breitenbach J, Schrof W, Neuman J, Confocal Raman Spectroscopy: analytical approach to solid dispersions, Pharm Res, 2007, 16:110913.

42. Guyot M, Fawaz F, Bildet J, Bonini F, Physicochemical characterization and dissolution of norfloxacin / cyclodextrin inclusion compounds and PEG solid dispersions, Int J Pharm, 1995, 12:53-63.

43. Sethia S, Sqillante E, Physicochemical characterization of solid dispersions of carbamazepine formulated by supercritical carbon dioxide and conventional solvent evaporation method, J Pharm Sci, 2002, 91:1948-57.

44. Liu R, edit or Water insoluble drug formulation, Library of Congress Cataloging-in-Publication Data, 2007.

45. Breitenbach J, Magerlein M, Melt Extruded Molecular Dispersion, In: Sellassie IG, Martin C, editors Pharmaceutical Extrusion Technology, Informa Health care, 2003, p. 246.

46. URL:http://www.pharmtech.findpharma.com/pharmtech/../article/...5 29177 